\title{
Présentation
}

\section{Arnaud Halloy}

\section{OpenEdition}

Journals

Édition électronique

URL : http://journals.openedition.org/span/1162

DOI : $10.4000 /$ span. 1162

ISSN : 2268-1558

\section{Éditeur}

École pratique des hautes études. Sciences humaines

\section{Édition imprimée}

Date de publication : 15 avril 2004

Pagination : 5-9

ISBN : 2-9090-3629-4

ISSN : 0294-7080

\section{Référence électronique}

Arnaud Halloy, «Présentation », Systèmes de pensée en Afrique noire [En ligne], 16 | 2004, mis en ligne le 06 novembre 2013, consulté le 22 septembre 2020. URL : http://journals.openedition.org/span/1162 ; DOI : https://doi.org/10.4000/span.1162 


\section{Présentation}

Arnaud Halloy

Université Libre de Bruxelles

L'ambition de ce numéro est à la fois ethnographique et théorique. Ethnographique puisque nous proposons une série de documents sur ce qu'il est convenu d'appeler les religions afro-américaines. Théorique puisque les diverses contributions rassemblées ici aboutissent, explicitement ou non selon les cas, à des propositions assez générales sur une notion visitée depuis longtemps par l'anthropologie sociale : le rituel. Au risque de simplifier un peu, on peut dire que les séquences d'actes plus ou moins formalisées que les anthropologues ont pris l'habitude de qualifier de rituelles ont fait l'objet de deux approches essentielles. Selon une première approche, le rituel est porteur d'une signification, il révèle les valeurs qui animent ceux qui l'accomplissent. En d'autres termes, il a quelque chose à dire, et on doit donc le lire, le déchiffrer, le décoder, l'interpréter. Selon une seconde approche, qui est peut-être plus en faveur aujourd'hui, le rituel ne dit pas, il fait. De sorte qu'il relèverait moins d'une sémantique que d'une sociologie de l'action. Cette seconde approche se scinde d'ailleurs à son tour en deux courants. On peut soit considérer que certains types d'actes, essentiellement différents des autres actes que nous accomplissons dans la vie sociale, doivent être qualifiés de « rituels ». L'acte rituel se distinguerait ainsi de l'acte technique, de l'action politique, etc. Ainsi, une version de 
ce courant tend à considérer que les actes rituels se caractériseraient par des dispositions intentionnelles spécifiques (Humphrey et Laidlaw, 1994.) Ou bien on peut considérer que la "ritualité ", si l'on peut dire, est un trait susceptible de colorer toutes sortes d'actions, éventuellement aux côtés d'autres traits. Il y aurait donc ainsi «du rituel " dans les salutations, dans la guerre, et peut-être même dans certains actes techniques.

Bien entendu, ces deux grandes approches cohabitent dans la plupart des études que la profession consacre au rituel. Même lorsqu'on considère le rituel comme une séquence d'actes, on peut aussi s'interroger sur les significations des symboles manipulés à l'occasion par ceux qui accomplissent ces actes. Et les études qui privilégient la seconde approche ne distinguent pas toujours les deux aspects qu'elle est susceptible de prendre. Le lecteur ne s'étonnera donc pas que, si nos contributions ont plutôt tendance à voir le rituel comme une séquence d'actes, elles ne sont pas toujours exemptes d'un certain souci interprétatif.

On ajoutera même que l'ambition ethnographique et l'ambition théorique qui ont été les nôtres ne sont pas disjointes elles non plus. En effet, les cultures afro-américaines se sont au cours de ces dernières années révélées être un laboratoire propice à l'étude de la circulation et des transformations, à travers le temps et l'espace, de savoirs et de savoir-faire propres à diverses populations (voir entre autres Stefania Capone $2001-2002$; 2004). Et nous ne pensons pas seulement ici aux mouvements d'un continent à l'autre auxquels se sont intéressées durant plus d'un siècle les études consacrées à l'" héritage africain " des populations noires aux Amériques. Car sur ce point, nous rejoignons le point de vue de S. W. Mintz (1970 : 11) :

Quite aside from the question of historical origins, the cultural resources of Afro-Americans and Afro-American culture are by no means limited to those elements or complexes that are probably African historically; such origins are far less significant than the continuing creative employment of forms, whatever their origins, and the symbolic usages imparted to them. 
C'est pourquoi, si les études rassemblées ici ont pleinement leur place dans une revue africaniste - car on ne peut tout de même nier qu'il y ait bien en la matière un héritage africain, même s'il est pour partie rêvé et reconstruit par les intéressés - elles engagent une réflexion plus générale sur tout un pan de la vie sociale; à savoir, cette " continuelle utilisation créative de formes ", et aussi bien de valeurs, de souvenirs, de significations, qu'on transmet, qu'on transforme et qu'on trahit parfois.

Patricia de Aquino a étudié la divination pratiquée quotidiennement au sein du Candomblé brésilien. Dans une belle et attentive étude de cas, elle fait notamment valoir que la tâche du devin est moins de prédire l'avenir que d'engager son consultant à envisager autrement le présent. Elle a pour cela suivi pas à pas une patiente venue consulter à cause d'un fils qui lui cause du souci, et avec lequel on voit que ses relations se modifient au fur et à mesure que les séances divinatoires se succèdent.

Philippe Jespers propose une analyse pragmatique d'une danse cubaine, la rumba. Plutôt que l'expression d'autre chose qu'elle-même (comme par exemple la relation homme/femme telle qu'on la retrouve dans la société cubaine), la rumba est prise pour ce qu'elle est, c'est-à-dire, comme il l'écrit en se souvenant d'Agamben, " une finalité sans fin [...], le processus, à jamais inabouti, de reconstruction (symbolique) de l'identité des sexes en interaction l'un avec l'autre ".

Arnaud Halloy s'est intéressé à un culte brésilien transplanté en Belgique. Les erês, divinités infantiles liées à ce culte présentent des traits parodiques qui incitent les néophytes à porter sur lui un regard distancié, grâce auquel ils sont mieux à même d'en intérioriser les conventions.

Dans une analyse d'une grande ambition comparative, qui prolonge et renouvelle celle de Victor Turner, José Jorge de Carvalho montre comment la violence et le « chaos "si présents dans certains cultes afro-brésiliens sont en tant que tels facteurs d'ordre et de paix. Pour le paraphraser d'un 
mot, si dionysiaque qu'il soit, un culte est parfois porteur de valeurs apolliniennes.

A propos de carnavals brésiliens et colombiens, Michel Agier revisite et questionne la notion d'identité. Les figures ambiguës, vaguement diaboliques, que mobilisent ces rituels carnavalesques, " ouvrent ", comme il le dit, "des chemins vers l'altérité »: je suis celui que je suis dans la mesure même où je suis un peu un autre...

Silvina Testa s'interroge sur le rôle respectif des hommes et des femmes dans les religions afro-cubaines, ce qui l'amène à rencontrer la figure de l'homosexuel. D'où une configuration à trois termes dont la hiérarchie est inaltérable en principe (d'abord l'homme homosexuel, puis l'homme hétérosexuel, et en dernier la femme), mais souvent contredite dans la réalité.

N.B. : Des divinités dont l'origine africaine est vraisemblablement la même se retrouvent avec des orthographes différentes selon qu'elles apparaissent en pays hispanophone ou au Brésil. Comme nous pensons justement que c'est leur usage et non leur origine supposée qui détermine l'identité de tel ou tel être cultuel, nous avons tenu à conserver les graphies locales, d'autant plus que l'inconvénient qui en résulte n'est pas insurmontable. 


\section{Références bibliographiques}

Capone, S.

2001-02 "La diffusion des religions afro-américaines en Europe ", Psychopathologie africaine, 31 (1), pp. 3-16.

2004 «A propos des notions de globalisation et de transnationalisation", Civilisations, 51 (1-2), pp 9-22.

Humphrey, C. \& J. Laidlaw

1994 The archetypal actions of ritual. A theory of ritual illustrated by the Jain rite of worship, Oxford, Clarendon Press.

Mintz, S.W.

1970 "Foreword", in N.E. Whitten et J. Szwed (dir.), Afro-American Anthropology. Contemporary Perspectives, New York, The Free Press, pp. 1-6. 
